\title{
Processing of Polymers with Supercritical Fluids
}

\author{
By Sacide Alsoy and J. Larry Duda*
}

\section{Introduction}

The removal of impurities, such as residual solvents, unreacted monomers, catalysts, and side-reaction products from polymers represents an important step in polymer processing. Conventional devolatilization techniques for the purification of polymers have limited effectiveness. Devolatilization with supercritical fluids, however, can enhance impurity removal by increasing the thermodynamic driving force and molecular diffusivity. The high pressures involved make this process a rather capital-intensive business. In certain applications, however, the additional processing cost can be offset by the benefits derived from high-specialty polymers that can be produced using this technology.

\section{Background}

The unique properties of supercritical fluids were reported almost one hundred years ago by observing that at high pressures gases, such as carbon dioxide and ethylene, can dissolve complex organic compounds and the dissolving power of these fluids is a strong function of pressure. However, the first commercial-scale supercritical processing of polymers was utilized in the 1950 s to manufacture billions of pounds of low-density polyethylene per year using supercritical ethylene. In this process, the compressed ethylene serves as both the monomer and the reaction solvent. Since that time, considerable effort has been expended to develop other commercial supercritical fluid processes. Particularly in recent years, the use of supercritical fluids to extract low-molecularweight compounds from polymer matrices has received much attention [1,7-11].

\section{Purification of Polymers With Supercritical Fluids}

Conventionally, polymers are purified by either vacuum, steam stripping or solvent extraction. These methods are not sufficient to reduce the residual contents to the required permissible levels. It has been reported in a USA patent that supercritical devolatilization has the potential to produce high-purity, high-quality products with lower energy costs [1].

[*] S. Alsoy, Izmir Institute of Technology, Gaziosmanpasa Bulv. No. 1635230, Cankaya-Izmir, Turkey; J. L. Duda, Center for the Study of Polymer Solvent Systems, Dept. of Chemical Engineering, The Pennsylvania State University, University Park, PA, 16802, USA.
A devolatilization process with supercritical fluids is significantly different from conventional devolatilization processes. In a typical supercritical devolatilization process, the first step is the compression of the fluid to elevated pressures above its critical pressure. The second stage is the extraction stage, in which the polymer is exposed to the supercritical fluid, it is swollen, and the free volume in the polymer is increased so that the diffusion of the impurities out of the polymer is enhanced. To illustrate this plasticization effect, the polystyrene/carbon dioxide/benzene system was chosen as a model system and the diffusivities of benzene and carbon dioxide were predicted from the Vrentas-Duda freevolume theory [2]. Fig. 1 shows that the diffusivity of benzene is increased by three orders of magnitude as the weight fraction of carbon dioxide increases from 0 to $10 \%$ in the mixture. The increase in the diffusivity of benzene is more pronounced than that of carbon dioxide which is shown in Fig. 2. In addition to the plasticization effect, the nonideal thermodynamic behavior of the supercritical fluid polymer solution can cause diffusioninduced convection which can influence the transport of impurities out of the polymer. Therefore, appropriate models to simulate the extraction stage of supercritical devolatilization processes should take into account the plasticization effect as well as the convective diffusion mechanism in mass transfer equation coupled with a suitable thermodynamic model to predict the partial specific volumes of components [3]. Fig. 3 illustrates the effect of diffusion-induced convection, caused by the nonideal thermodynamic behavior of the polystyrene/ carbon dioxide/benzene system, on the benzene removal rate. According to this figure, the loss of benzene in polystyrene during the extraction with supercritical carbon dioxide is enhanced by the inclusion of the volume change on mixing. In the final stage of the supercritical devolatilization process, the swollen polymer phase is depressurized and gradually relaxes and returns to, or at least approaches, its original volume. During the swelling-deswelling of the polymers, significant changes occur in the polymer morphology, especially in glassy polymers. Hong and coworkers [4] found that the free-volume fraction is always higher during depressurization than it is under pressurization. If the relaxation time of the polymer to the unswollen state is slow during the depressurization stage, then some of the volume occupied by the absorbed supercritical fluid becomes available as free volume for the transport of the impurity. Predictions from the free-volume theory indicate that the diffusivity of benzene in unrelaxed polystyrene at $40{ }^{\circ} \mathrm{C}$ after 10 wt.- $\%$ carbon dioxide has desorbed can be as high as 3.7 $\times 10^{-4} \mathrm{~cm}^{2} / \mathrm{sec}$ if all the volume occupied by carbon dioxide becomes available to the diffusing benzene. This value represents the maximum diffusivity for the impurity, which would decrease as the polymer consolidates and relaxes toward its equilibrium state. It is an enormous diffusivity compared to 
the estimated diffusivity of $3 \times 10^{-13} \mathrm{~cm}^{2} / \mathrm{sec}$ [5] for benzene in conventional glassy polystyrene at $40^{\prime} \mathrm{C}$ or even the estimated value of $6 \times 10^{-9} \mathrm{~cm}^{2} / \mathrm{sec}$ for benzene in polystyrene plasticized with 10 wt.-\% carbon dioxide. This mechanism of rapid impurity extraction during the depressurization step is supported by the observations of Sasaki and coworkers [6] which are illustrated in Fig. 4. This figure shows that, if polystyrene containing $4.5 \mathrm{wt}$. \% benzene is exposed to the supercritical carbon dioxide at $40^{\circ} \mathrm{C}$ for $1.5 \mathrm{~h}$, the concentration of benzene is reduced to approximately $3.5 \mathrm{wt} .-\%$. However, during the very short depressurization step, the concentration of benzene is reduced to $3 \%$. The authors observed a similar trend for the polyvinyl acetate/benzene system at the same temperature.

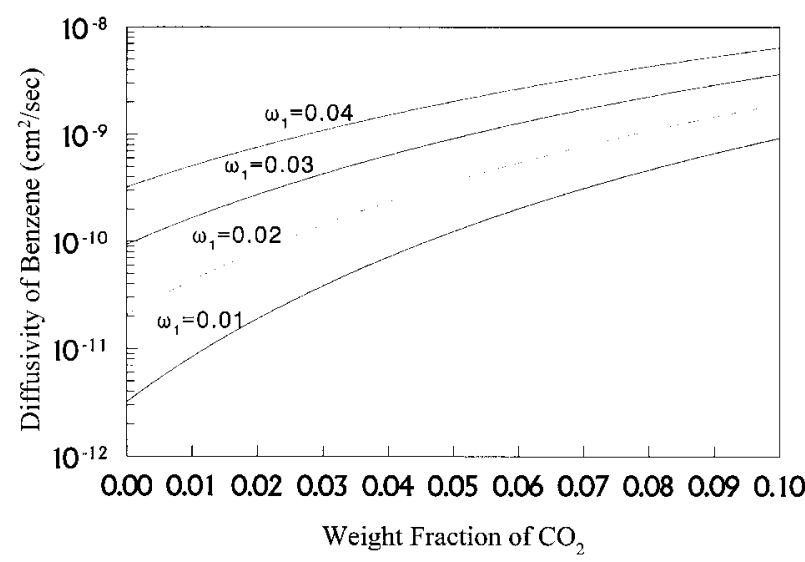

Figure 1. Self-diffusion coefficient of benzene as a function of the carbon dioxide composition in $\mathrm{PS} /$ benzene/ $\mathrm{CO}_{2}$ ternary mixture where $\mathrm{T}_{1}$ refers to the weight fraction of benzene.

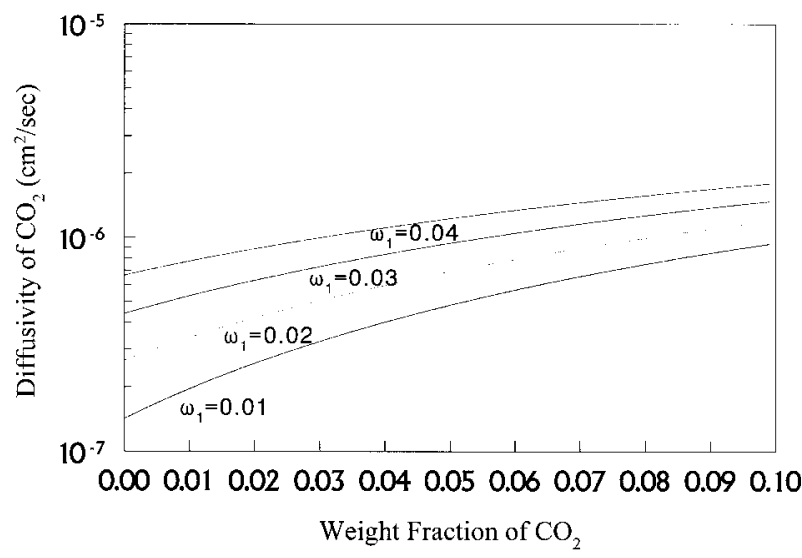

Figure 2. Self-diffusion coefficient of carbon dioxide as a function of the weigh fraction of carbon dioxide in $\mathrm{PS} /$ benzene $/ \mathrm{CO}_{2}$ ternary mixture where $\mathrm{T}_{1}$ refers to the weight fraction of benzene.

\section{Conclusion}

In conventional supercritical extraction, the high solubility of impurities in the supercritical fluid can enhance the thermodynamic driving force for extraction. In addition, the

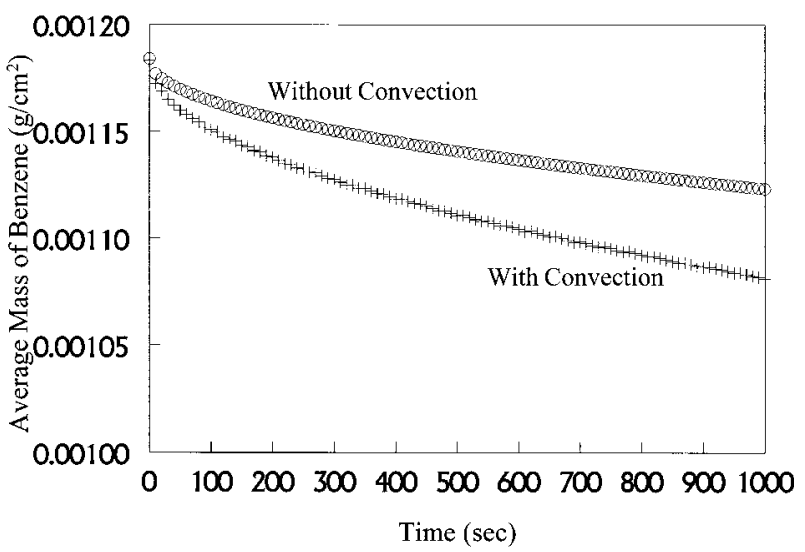

Figure 3. Mass of benzene per unit area of the polymer film as a function of time during the absorption of the carbon dioxide.

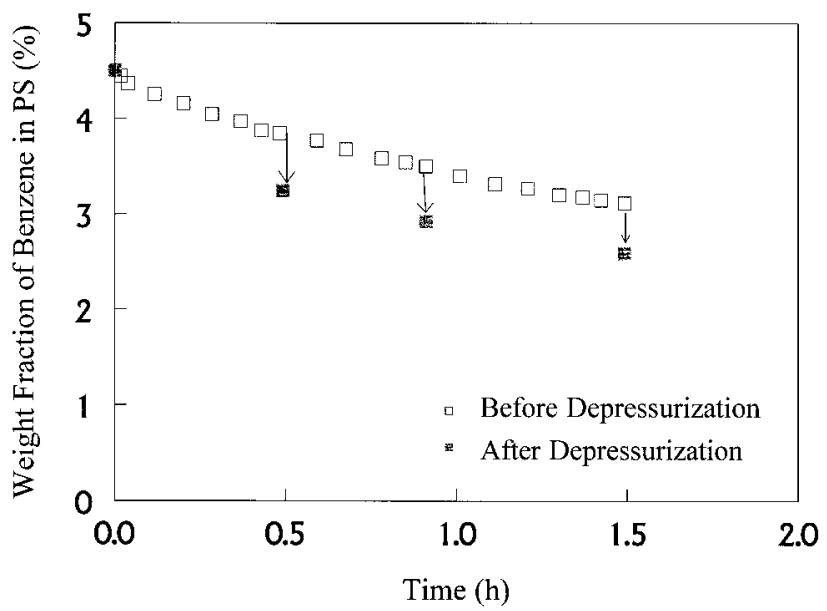

Figure 4. Weight fraction of benzene in the polystyrene phase as a function of time in the extractor. Data taken from the study of Sasaki et al. (1990) at a pressure of $7.95 \mathrm{MPa}$ and $40{ }^{\circ} \mathrm{C}$.

high solubility of the supercritical fluid in the polymer can swell the polymer and can increase the mobility of impurities. In addition to these two effects, there are two other phenomena which can be important in supercritical devolatilization of glassy polymers. During the extraction stage, the nonideal volumetric behavior of supercritical fluids in polymers can cause convection induced by diffusion which can influence the transport of impurities out of the polymer. Furthermore, during the depressurization step, the coupling between mass transfer and the polymer relaxation can be important. The free-volume theory indicates that diffusion of the impurity in the unrelaxed polymer can be several orders of magnitude higher than the diffusivity that occurs in the plasticized polymer during the extraction stage. The most significant conclusion resulting from our work is that the mass transferred during the very short depressurization step may be the dominant mechanism in supercritical fluid devolatilization of polymers. This conclusion is supported by the experimental studies of Sasaki and coworkers [6] and leads to important implications for the optimum design and operation of the devolatilization of polymers with supercritical fluids. This proposed mechanism suggests that impurity removal can be 
facilitated by shortening the time of the depressurization step and lowering the operating temperature of the process. Both of these modifications will enhance the mass transfer associated with diffusion in an unrelaxed glassy polymer phase. Furthermore, for efficient devolatilization, the time of exposure of the polymer phase to the supercritical fluid can be significantly reduced by designing processes to exploit volatility removal during the depressurization phase of the operation.

Received: May 11, 1999 [RN 009]

\section{References}

[1] Copelin, H. B., Method For Reducing Oligomeric Cyclic Ether Content of a Polymerizate, US Patent No. 4.306.058 (1981).

[2] Duda, J. L.; Vrentas, J. S.; Liu, Ju and H. T., Prediction of Diffusion Coefficients for Polymer Solvent Systems, AIChE J. 28 (1982) 285.

[3] Alsoy, S.; Duda, J. L., Supercritical Devolatilization of Polymers, AIChEJ. 44 (1998) 582.
[4] Hong, X.; Yuan, J. P.; Huang, C. M.; Cao, H.; Jean, Y. C., Gas Permeation and Free-Volume Properties Probed by Positron Annihilation, Polymeric Materials Science and Engineering, Proceedings of the American Chemical Society Division of Polymeric Materials, Spring Meeting, 76, 346, April 1997, San Francisco.

[5] Kosfeld, R.; Zumkley, L., Mobility of Small Molecules in Polymer Systems," Ber. Bunsenges. Phys. Chem. 83 (1979) 392.

[6] Sasaki, M.; Takishima, S.; Masuoka, H., Supercritical Extraction of Benzene in Poly(vinyl acetate) and Polystyrene (Part 2), Sekiyu Gakkaishi 33 (1990) 304.

[7] Krukonis, V., Processing of Polymers with Supercritical Fluids, Polymer News 11 (1985) 7.

[8] Jarzebski, A. B.; Lachowski, A. I.; Solich, L. M.; Wenzel, H., Purification of the Biologically Active Copolymers of Maleic Anhyride by Supercritical Fluid Extraction, Makromol. Chem. Rapid Commun. 11 (1990) 349.

[9] Scholsky, K. M., Process Polymers with Supercritical Fluids, Chemtech. 17, 750 (1987).

[10] Venema, A.; Van De Ven, H. J. F. M.; Davis, F.; Sandra, P., Supercritical Fluid Extraction of Nylon - An Investigation into the Factors Affecting the Efficieny of Extraction of Caprolactam and its Oligomers, J. High Res. Chrom, 16 (1993) 522.

[11] Yilgor, I.; McGrath, J. E., Supercritical Fluids - Novel Supercritical Fluid Techniques for Polymer Fractionation and Purification, Polymer Bulletin 12 (1984) 491. 